
allemande

47-2 | 2015

Retour sur le modèle Rhénan : humanisme, capitalisme et métropolisation | Patrimonialisation du passé « allemand » en Europe centrale après 1990

\title{
Konstruierte Heimat. Grenzziehung durch Tracht am Beispiel von Martin Dušeks Dokumentarfilm Mein kroj
}

Christina Köck

\section{OpenEdition}

Journals

\section{Édition électronique}

URL : https://journals.openedition.org/allemagne/286

DOI : 10.4000/allemagne.286

ISSN : 2605-7913

Éditeur

Société d'études allemandes

\section{Édition imprimée}

Date de publication : 16 décembre 2015

Pagination : 473-488

ISSN : 0035-0974

\section{Référence électronique}

Christina Köck, „Konstruierte Heimat. Grenzziehung durch Tracht am Beispiel von Martin Dušeks Dokumentarfilm Mein kroj", Revue d'Allemagne et des pays de langue allemande [Online], 47-2 | 2015, Online erschienen am: 13 Dezember 2017, abgerufen am 18 Mai 2021. URL: http:// journals.openedition.org/allemagne/286 ; DOI: https://doi.org/10.4000/allemagne.286 


\section{Konstruierte Heimat. Grenzziehung durch Tracht am Beispiel von Martin Dušeks Dokumentarfilm Mein kroj}

\section{- Christina Köck*}

Der Modetrend Tracht dauert seit Jahren an: Dirndl und Lederhosen sind längst aus der deutschtümelnden Ecke herausgeholt worden, in der sie sich vor allem nach ihrer Instrumentalisierung durch die Nationalsozialisten befanden. Vor allem im süddeutschen und österreichischen Raum werden sie mit postmoderner Lust an der Verkleidung bei verschiedensten Gelegenheiten - allen voran natürlich beim Oktoberfest - getragen. Doch sind die Kleider noch so schrill und deren Trägerinnen und Träger noch so international: Tracht bleibt ein starkes Zeichen materieller Kultur, das regionale oder gar nationale Identität (mit) symbolisiert. Gerade mit dem Trend zum Dirndl allgemein ist „echte“ Tracht in Mode gekommen. Kleidung, die für eine bestimmte Region und/oder Personengruppe kreiert wird und - sicher auch aus Marketinggründen - für diese stehen soll: Tradition boomt, vom Niederösterreich-Dirndl über die Wechsellandtracht bis zum Jungbauernanzug.

Bei Vertriebenen aus ehemals von Deutschsprachigen besiedelten Gebieten in Zentral- und Osteuropa hat das Tragen von Tracht einen stark politischen Hintergrund, vor allem bei den - ich benütze bewusst die nicht unproblematische Eigenbezeichnung - „Sudetendeutschen“.

Vor allem aufgrund des symbolischen Einsatzes als deutsche Uniform im Zuge der Heim ins Reich - Bewegung ist sie bei der deutschsprachigen Minderheit im heutigen Tschechien mit einer bestimmten Ideologie aufgeladen, dazu im zweiten Teil des Aufsatzes mehr. Bis heute werden die „althergebrachten“ Kleider auf Treffen von Vertriebenen und bzw. vor allem von deren Nachkommen kaum spielerisch-postmodern als Verkleidung genutzt. Sie dienen vielmehr als starkes Zeichen der Identifizierung mit dem verlorenen Böhmen und Mähren. Regionen, die, wenn auch keine

\footnotetext{
* Maître de langue, lectrice OeAD, Université de Strasbourg.
} 
„Wiedergewinnung“ seitens der Landsmannschaft mehr angestrebt wird ${ }^{(1)}$, für diese doch weiterhin als verlorene Heimat, als Sehnsuchtsort stehen. Sichtbar wird dies zum Beispiel am alljährlichen Sudetendeutschen Tag in Augsburg, an dem „traditionell sudetendeutsche“ Tracht getragen wird.

In Mein kroj von Martin Dušek wird ein solcher Sudetendeutscher Tag und die Beziehung von dessen Besucherinnen und Besuchern zu Tracht zum Thema gemacht und karikiert, weshalb der Film im ersten Teil des Artikels konkret vorgestellt werden soll. Danach wird die These „Abgrenzung konstruiert Tracht konstruiert Abgrenzung“ geprüft. Anhand der Beispiele aus dem Film bzw. der Tracht im „Sudetenland“ und dem Fall Salzburg in den 1930er Jahren im zweiten und dem Trachtenboom im süddeutschen Sprachraum im 21. Jahrhundert im dritten Abschnitt des Texts möchte ich die engen Verflechtungen zwischen der ständigen Neuerfindung von Tracht und deren Ausgrenzungsmechanismen aufzeigen und in diesem Kontext auch versuchen, die Frage zu beantworten, ob das Dirndl heute wirklich zum völlig ideologiefreien Modetrend geworden ist.

\section{Martin Dušeks Film Mein kroj: Ein Eindringling auf dem Sudetendeutschen Tag 2011}

Der 1978 geborene, mehrfach preisgekrönte Filmemacher Martin Dušek beschäftigt sich in seinen Werken immer wieder mit tschechischer Zeitgeschichte und den Konsequenzen großer Politik auf von ihm gezeigte kleine Leute. Wiederholt hat er sich mit den ehemals von Deutschsprachigen besiedelten Gebieten des heutigen Tschechien und der Frage der Viktimisierung bzw. mit Schuldzuweisungen auf beiden Seiten der Grenze befasst. Sein Film Český žurnál: The Resounded Village (2013) zum Beispiel thematisiert den Umgang mit dem 1942 als Vergeltung auf das Attentat auf Reinhard Heydrich zerstörten Ort Lidice im kommunistischen Regime sowie heute ${ }^{(2)}$, in Koblakum vzhlizime (2014, engl. Titel Into the clouds we gaze) beschäftigt er sich mit der schwierigen ökonomischen Situation in Nordböhmen. Dušek zeigt die Protagonisten in seinen Dokumentarfilmen in ihrer Widersprüchlichkeit, wodurch seinen Filmen Authentizität, gleichzeitig aber auch Unterhaltsamkeit verliehen wird. Auch Mein kroj ist keine Schwarz-Weiß-Zeichnung, sondern eine kritische, wenn auch provokante Annäherung an das Thema Tracht auf beiden Seiten der Grenze. Im Folgenden sei der 2011 erschienene Film ${ }^{(3)}$, der im deutschsprachigen Fernsehen noch nicht gezeigt wurde, umrissen:

Dušek, Protagonist in seinem eigenen Film, stilisiert sich als sympathisch-naiver „Schelm“ im besten Sinn des Wortes: Er hätte schon als Kind gern eine Tracht gehabt, weil er bei Reisen nach Südmähren immer so begeistert von den Kleidern der Menschen gewesen sei. Leider habe es in der kommunistischen Tschechoslowakei nur die

1 Siehe dazu den ohne Angabe eines Autors veröffentlichten Artikel „Landsmannschaft verzichtet auf ,Wiedergewinnung der Heimat““, Die Zeit vom 1. März 2015, http://www.zeit.de/politik/deutschland/ 2015-03/sudetendeutsche-landsmannschaft-heimat (Mai 2015).

2 Gespräch mit Martin Dušek am 4. November 2013.

3 Martin DušEK, Mein kroj. Dokumentarfilm, Prag, 2011, http://www.ceskatelevize.cz/porady/ 10318067925-mein-kroj/ (Mai 2015). 
Uniform der örtlichen Pioniere gegeben, deshalb macht er sich nun auf die Suche nach der Tracht seiner Heimatregion. Seine Heimatstadt Česká Lípa, - auf Deutsch Böhmisch Leipa in Nordböhmen -, war vor 1945 zum größten Teil von Deutschen bewohnt. Im örtlichen Museum stößt Dušek nun auf eine Tracht, die sich in Gesprächen mit der Bürgermeisterin von Česká Lípa und einem Historiker schnell als im Jahr 1947 kreiert entpuppt. Dušek ist enttäuscht: „Diese Tracht hat mir überhaupt nicht zugesagt. Eine willkürliche Tracht ist halt nichts, womit ich mich identifizieren kann“(4). Im Interview mit Radio Prag äußerte er sich später eindeutiger über das Museumsstück: „Es ist ein unsinniger Hokuspokus aus verschiedensten, miteinander nicht zusammenhängenden Elementen “(5).

Gemeinsam mit einer lokalen Schönheitskönigin, die die Tracht aus dem Museum trägt, fragt Dušek Passantinnen und Passanten danach, ob sie die Kleidung wiedererkennen - und stößt auf überraschte, ungläubige Gesichter; niemandem ist sie vertraut.

Dušek, der nach eigenen Angaben „teilweise auch Sudetendeutscher ist“ - sein Großvater war halb Deutscher, hatte sich aber zur tschechischen Volksgruppe bekannt - ist

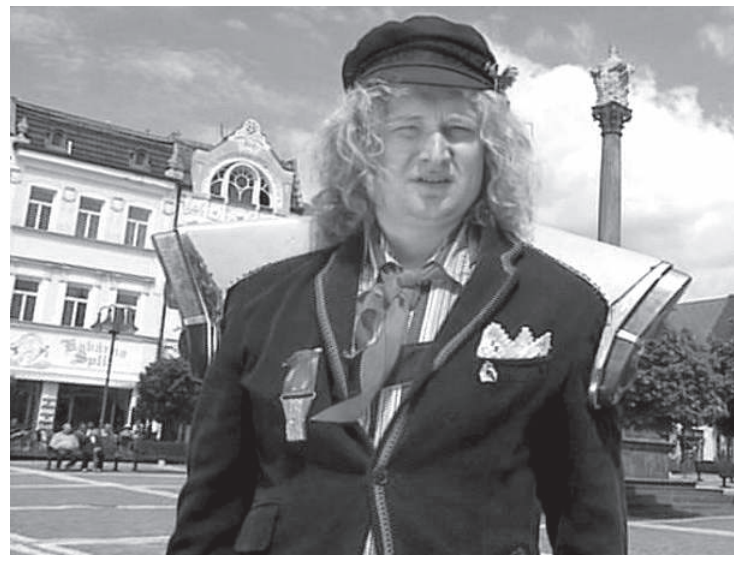

Screenshot aus Mein Kroj: Martin Dušeks selbstgebastelte Tracht (mit freundlicher Genehmigung von Česká televize) enttäuscht. Dann jedoch hat er die zündende Idee: Er beschließt, seine ganz persönliche Tracht, seinen „kroj“ - das tschechische Wort für Tracht - zu kreieren. In diese eingearbeitet sind Familienerinnerungen, besonders jene an seine Großeltern wie die Lederhose seines Großvaters, eine Masaryk-Mütze, eine Spitzendecke seiner Großmutter und, definitiv das Highlight, die Blinker des alten Škoda Oktavia seines Großvaters. Die Objekte sind allesamt alt und haben in der und für die Familie eine bestimmte Bedeutung, sodass diese individuelle Tracht durchaus als traditionell im Sinne von überliefert oder übergeben bezeichnet werden kann.

Doch er ist mit seiner Kreation nicht am Ziel, die Suche nach der ursprünglichen, der alten Tracht aus seiner Heimat lässt ihn nicht los. Unbedingt möchte er frühere Bewohnerinnen und Bewohner seiner Heimatstadt finden, weshalb er sich auf den Weg zum 62. Sudetendeutschen Tag in Augsburg macht, wo er hofft, viele ehemals in Böhmisch Leipa Ansässige zu finden, die ihm mehr über die Tracht dort erzählen können. Gekleidet ist er natürlich in seiner Tracht - und wird prompt von Hanni Köglsperger, der Geschäftsführerin der Sudetendeutschen Landsmannschaft, skeptisch empfangen.

5 Marco Zimmermann, „Ein satirischer Beitrag zur Identitätssuche: Der Dokumentarfilm Mein kroj“, Radio Praha, 2012, http://www.radio.cz/de/rubrik/kultur/ein-satirischer-beitrag-zuridentitaetssuche-der-dokumentarfilm-mein-kroj (Mai 2015). 
Die beiden führen eine Diskussion über die Frage „Was ist Tracht?“, die im Folgenden transkribiert wiedergegeben wird:

„Köglsperger: Ist das eine Tracht, die Sie tragen?

Dušek: Ja, das ist mein[e] Tracht.

Köglsperger: Aha... und ist die landschaftsbezogen, aus einem Bereich in der tschechischen

Republik oder...?

Dušek: Nein, das ist mein[e] eigene Tracht.

Köglsperger: Sie haben für sich diese Tracht ausgewählt.

Dušek: Ausgedacht, ja.

Köglsperger: Würden Sie mir sagen, was das darstellt? Würd' mich interessieren...

Dušek: Ja! [...]

Köglsperger: Wenn Sie eine Tracht haben, das wär' wunderschön. Tracht[en] ist was Wunderschönes. Unsere Gäste haben sehr viele Trachten an. Aber ich finde, das ist jetzt keine Tracht. Es ist ein Konstrukt, das Sie sich zusammengestellt haben.

Dušek: Was ist Tracht?

Köglsperger [mit Unterstützung eines Herren im Hintergrund]: Tracht ist was Historisches, über Jahrhunderte, aus einer typischen Gegend, das hat schon der Ururgroßvater gehabt.

Dušek: [...] und wann startet das, Tracht?

Herr im Hintergrund: Da könnte man auch genauso fragen, wann startet Geschichte. Das ist Geschichte“(6).

Dušeks Kleidung erfüllt also offenbar nicht die klassischen Kriterien von Tracht: Weder ist sie immer schon dagewesen oder tut so, noch ist sie landschaftsbezogen, wird also von einem Kollektiv getragen: Sie ist ein Puzzle aus Familienerinnerungen.

Vorerst weiter im Film: Dušek geht zum Sudetendeutschen Tag, amüsiert sich, tanzt mit einer Frau in Egerländer Tracht und findet endlich eine Dame aus Böhmisch Leipa, mit der er über Kleidungstraditionen in der Region spricht. Sie erzählt ihm, dass Tracht in der Region nicht üblich gewesen sei und dass durchaus nicht alle Sudetendeutschen Tracht getragen hätten. In das Gespräch platzt Jan Šinágl, ein tschechischer Aktivist, der sich für die Rechte der vertriebenen Sudetendeutschen einsetzt, und bezichtigt Dušek, sich über die Dame lustig zu machen und im tschechischen Fernsehen die Sudetendeutsche Landsmannschaft schlechtmachen zu wollen. Gemeinsam beschwichtigen ihn Dušek und die - sehr sympathische - ältere Dame: Sie sprächen nicht über Politik, Tracht sei ja nicht politisch $^{(7)}$.

Der Film nimmt eine unschöne Wende: Der Trachten-Fan Dušek wird wegen Hausfriedensbruchs vom Gelände entfernt und unter seinem Geschrei, er werde vertrieben, verhaftet und auf die Polizeistation gebracht.

Um es gleich vorweg zu nehmen: Die Anzeige wurde noch während des Tages fallengelassen und Dušek wurde 2013 sogar von der Jugend der Sudetendeutschen Landsmannschaft auf den Sudetendeutschen Tag eingeladen. Er sollte dort an einer Diskussion zum Thema „Geschichte - ein Monopol der Alten?" teilnehmen und sagte unter der Bedingung zu, in seiner Tracht auftreten zu dürfen, was ihm zugestanden wurde. Letztlich scheiterte der Besuch an terminlichen Problemen, auf der Homepage

6 M. DušEK, Mein Kroj (Anm. 3), 09:46-11:44.

7 Ebd., 18:40-20:19. 
des 64. Sudetendeutschen Tages wurde er als Teilnehmer aufgeführt. Diese Reaktion scheint ein Hinweis auf eine bestimmte Öffnung bei der Sudetendeutschen Landsmannschaft zu sein, die in den letzten Jahren stattfindet. Auch in Česká Lípa kam es nicht zu Aufregungen im Zusammenhang mit dem Film, im Gegenteil: Die Christdemokraten in der Stadt wollten ihn sogar als Kandidaten für die Wahl zum Stadtrat haben. Seinen kroj wollten sie allerdings nicht im Museum ausstellen - der befindet sich jetzt in der Divus Gallery in London.

In einer Pressekonferenz bei der Premiere des Films meinte der Filmemacher zu seinem kroj: „Die Tracht wirkt schon verrückt mit den Lampen vom Oktavia und so, aber ich habe mich darin gut gefühlt. Auf einmal trägt man etwas, was einen repräsentiert. Ich würde daher jedem empfehlen, sich seine eigene Tracht zusammenzustellen - man wird sich besser fühlen“(8).

Martin Dušek vollführt mit seiner Satire ein doppeltes Spiel:

Einerseits erfindet er selbst eine Tracht und persifliert damit die Erfindung und ständige Erneuerung von Tracht als althergebrachte Tradition im Sinne von Hobsbawm/ Ranger.

Der Film wimmelt auch darüber hinaus von als sehr alt verkauftem Brauchtum, über dessen Wurzeln sich die ProtagonistInnen nicht im Klaren sind und das sie, wie der Herr im Film, als „Geschichte“ bzw. „immer schon dagewesen“ klassifizieren. Abgesehen von den Trachten platziert Dušek unter anderem das „traditionelle Gebäck“ aus Česká Lípa, Trdelnik, prominent im Film. Eine Speise, die in verschiedenen Gebieten als ganz typisch für die Heimat verkauft wird; der tatsächliche Herkunftsort - ob Siebenbürgen, die Slowakei oder doch Ungarn - kann an anderer Stelle geklärt werden.

Andererseits kreiert Dušek ausdrücklich eine Tracht für sich persönlich, wodurch er den kollektiven Charakter dieser Kleidungsform pervertiert. Im Gegensatz zum Dirndl, das im allgemeinen Sprachgebrauch noch häufig als regional unabhängiges Kleidungsstück klassifiziert wird, hat ja Tracht im engeren Sinn eine stark kollektive Komponente. Auch wenn diese ursprünglich vor allem daher stammt, dass eine Schneiderin in einer bestimmten Gegend mit bestimmten Materialien arbeitete, die sie eben zur Verfügung hatte, wurden gerade im heutigen Tschechien sowohl bei der deutschsprachigen als auch bei der tschechischsprachigen Bevölkerung in der ersten Hälfte des 20. Jahrhunderts Erneuerungsbewegungen gestartet, die die Tracht als nationales Identitätsmerkmal herausstellen sollten. Gerade das Egerland erlebte eine massive Erneuerungsbewegung für die „Egerländer Tracht“, die später sogar als Uniform der Sudetendeutschen im Volkstumskampf eingesetzt wurde ${ }^{(9)}$. So ist es natürlich kein Zufall, dass der Filmemacher ausgerechnet eine Frau in Egerländer Tracht zum Tanz bittet und deren freundlich-naive Unwissenheit zur Geschichte ihrer Kleidung aufzeigt.

Ich denke, dass die Erfindung der Tradition und der exklusive Charakter gerade von Tracht sehr eng zusammenspielen. Anhand der bereits zu Beginn genannten These

8 M. ZimmermanN, „Ein satirischer Beitrag zur Identitätssuche“ (Anm. 5).

9 Cf. Elisabeth Fendl, „Die Politisierung der Tracht im Egerland des frühen 20. Jahrhunderts. Eine Projektskizze“, in: Heidrun Alzheimer et al. (Hg.), Jahrbuch für europäische Ethnologie, Paderborn u. a., 2009, S. 69-80. 
„Abgrenzung konstruiert Tracht konstruiert Abgrenzung“ - frei nach Ulrich Wehler, der Ähnliches über den Nationalismus und die Nation schreibt ${ }^{(10)}$, möchte ich mich beiden Phänomenen annähern.

\section{Eine nationalistisch motivierte Erfindung von Tradition}

Tracht „war schon immer“, ist „etwas natürlich Gewachsenes“

Die moderne Volkskunde oder besser, europäische Ethnologie würde diese im Film, aber auch davon unabhängig immer wieder geäußerte Aussage natürlich zurückweisen. Bis auf wenige unbelehrbare Trachtenerneuerinnen und -erneuerer besteht weitgehend Konsens, dass Trachten vor allem im 19. Jahrhundert zur regionalen und/ oder nationalen Identitätsbildung verwendet wurden. Bis heute leben sie von einer „simulierten Authentizität", wie es Stefan Hirsch, Bezirksheimatpfleger in Bayern, nennt ${ }^{(11)}$.

Kleidung ist ein wichtiger Bestandteil der materiellen Kultur und gerade die Tracht spielt eine herausragende Rolle für die Schaffung einer Gemeinschaft, ist sie doch fast wie eine Uniform Symbol für die Zugehörigkeit - in diesem Fall die regionale/ nationale ${ }^{(12)}$, jene als frühere deutschsprachige Minderheit im heutigen Tschechien. Mit der Bildung der europäischen Nationalstaaten wurden Trachten sukzessive immer mehr zu einem solchen Identifikationszeichen, Tobias Weger erklärt dieses Phänomen als „Entkontextualisierung traditioneller Gewandelemente und ihre Umdeutung zu rein folkloristischen Bekleidungen “(13). Dies kritisiert unter anderem der österreichische Schriftsteller Peter Rosegger im Jahr 1894, wenn er sich über die „Maskerade“ und „künstliche Wiedereinführung des Volksthums“ mokiert ${ }^{(14)}$. Der bekannte „Heimatdichter" ist einer unter vielen, die die Erneuerungsbewegungen der Tracht negativ bewerteten. Der Streit um Echtheit oder „Maskerade“ - interessant, dass Rosegger und ein Herr in Dušeks Film sogar das gleiche Wort verwenden - ist also ein alter. Fritz Rumpf schreibt schon 1905, dass die Kontroverse um Tracht gang und gäbe sei ${ }^{(15)}$.

Auf den Punkt gebracht: Erneuerung bringt Abgrenzung: „Echtes“ von „Maskerade“, Trachtenregion A von Trachtenregion B.

\section{Tracht als Bekenntnis zum „Deutschtum“}

Die erste Hälfte des 20. Jahrhunderts ist gekennzeichnet durch eine immer stärkere Beschäftigung mit dem Thema. So kommt es in den 20er Jahren vor allem im alpenländischen Raum zu einem Dirndl-Boom, in den 30er Jahren wird die Tracht, die im Film von deren Trägerinnen und Trägern vermutlich aus Unwissenheit als ganz unpolitisch dargestellt wird, immer deutlicher mit Ideologie aufgeladen. Gerade die

10 Hans-Ulrich Wehle, Nationalismus. Geschichte, Formen, Folgen, München, 2007, S. 13.

11 Heidi Sснмідт, „Was Sie schon immer über Tracht wissen wollten“. Interview mit Stefan Hirsch, Alpenmagazin. o. J, http://www.alpenmagazin.org/index.php/gesellschaft/100-deutschland26/702was-sie-schon-immer-ueber-trachten-wissen-wollten (Mai 2015).

14 Peter Rosegger, „Künstliches Volksthum“, Heimgarten, 19 (1895), S. 69. 
„Sudetendeutschen“ spielen im „Volkstumskampf“, im Einsatz von Tracht als „deutschem“ Symbol eine entscheidende Rolle. So schreibt der Volkskundler Josef Hanika, wohl der entscheidende Protagonist für die deutsche „Trachtenerneuerungsbewegung" in der Tschechoslowakei, 1939:

„Heute wird das Tragen der Tracht aus einer unbewußten Sitte zu einem bewußten Bekenntnis der Heimat- und Volksverbundenheit. Sie ist nicht nur Ausdruck der Gemeinschaft: Tragen der Tracht erweckt und vertieft den Gemeinschaftsgeist, schließt nach innen zusammen und nach außen ab, stärkt den Willen zur Behauptung, betont den Vollanspruch auf den Heimatboden gegen fremden Zugriff“(16).

Damit ist der performative Akt des Sich-Kleidens klar definiert. Wie Tobias Weger in einem Artikel über die „Völkische“ Wissenschaft aufgezeigt hat, führte Josef Hanika sein politisches Engagement für die Tracht auch nach 1945 fort - lediglich die Ziele waren unterschiedlich: So habe Hanika vor 1938 eine „folkloristische Uniformierung“ nach dem Motto Heim ins Reich intendiert. Nach 1945 sei die Trachtenpflege nahtlos fortgeführt und so im Zeichen einer, so Weger, „für die Revision der alliierten Nachkriegsbestimmungen [...] kämpfenden Sudetendeutschen Landsmannschaft“ gestanden $^{(17)}$.

Im Elsass lobt 1942 der Volkskundler Rudi Keller die „Grenzdeutschen“, die auch ihre Kleidung im „Volkstumskampf“ einsetzen, mit vorgetäuschter Naivität:

„Nicht wenige Volksgenossen fragten sich [...] in den letzten Jahren: Warum gilt der Haß des Dollfuß- und Schuschniggsystems in Deutsch-Österreich einem Bestandteil der deutschen Volkstracht, den weißen Strümpfen? [...] Warum verfolgen auch die Tschechen die weißen Strümpfe und das Dirndlkleid der Sudetendeutschen? Warum legt während des Einmarsches deutscher Truppen im Sudetenland die sudetendeutsche Bevölkerung spontan die bisher verfemte Tracht an?“(18)

$\mathrm{Zu}$ diesem Zitat muss angemerkt werden, dass die weißen Strümpfe zur kurzen Lederhose ab 1934 als Erkennungssymbol für illegale Nationalsozialisten in Salzburg dienten ${ }^{(19)}$, womit die scheinbare Frage nach dem Warum schnell geklärt ist. Sowohl die „sudetendeutsche Volksgemeinschaft“ als auch die Salzburger Illegalen hatten also ihre Kleidung bewusst als politisches Kampfsymbol eingesetzt, als „Bekenntnis zu [deutschem!] Stamm, Volk und Heimatboden “(20). Gerade der Text zeigt allerdings eine Problematik auf, die bereits im Zusammenhang mit dem Gespräch mit der Dame angerissen wurde, die die Egerländer Tracht trug: Dass diese Kleidung im Kulturkampf bewusst als Symbol eingesetzt wurde, ist evident. Weniger bekannt ist, wie groß das Wissen über die starke ideologische Bedeutung und das Bewusstsein über die hohe politische Konnotation bei der breiten Masse an Trägerinnen und Trägern

16 Josef Hanika, „Neugestaltung der Trachten im Sudetengau“, Deutsche Volkskunde, 3 (1939), S. 234240, hier S. 235.

17 Tobias Weger, „Völkische‘ Wissenschaft zwischen Prag, Eger und München. Das Beispiel Josef Hanika“, in: Christiane Brenner et al. (Hg.), Geschichtsschreibung zu den böhmischen Ländern im 20. Jahrhundert, München, 2006, S. 177-208, hier S. $204 \mathrm{f}$.

18 Rudi Keller, Tracht am Oberrhein, Strasbourg, 1942, S. 11.

19 Cf. ebd., S. 187.

20 E. FendL, „Die Politisierung der Tracht im Egerland des frühen 20. Jahrhunderts“ (Anm. 9), S. 75. 
tatsächlich war. Heute ist es, wie aufgezeigt wurde, definitiv nicht sehr ausgeprägt bzw. häufig diffus.

\section{Modetrend Tracht}

Gleichzeitig mit der Aufladung mit Bedeutung erlebte die Tracht in bestimmt nicht völkisch-rassistischen Kreisen ihren ersten großen Aufschwung als Modephänomen: Sie boomte bei der „High Society“, wie Ulrike Kammerhofer-Aggermann am Beispiel Salzburg zeigt ${ }^{(21)}$. Max Reinhardt inszenierte 1933 seinen „Faust“ bei den Salzburger Festspielen in Tracht, Dirndl wurden von Stars wie Marlene Dietrich getragen und auch zum Beispiel in die USA exportiert. Das Tragen der Tracht war also ein Modephänomen, das Dirndl galt als chic - ein Trend, der mit jenem zur Jahrtausendwende durchaus vergleichbar ist. Stefan Zweig, der, als er in Salzburg lebte, fast ausschließlich Trachtenkleidung trug, kann ein Hang zum völkisch-rassistischen Deutschtümler zweifellos abgesprochen werden, und auch die anderen Genannten sind über diesen Verdacht erhaben. Steht damit neben der Uniformierung ein frühes Spiel mit der Tradition, wurde die Kleidung ideologiefrei verwendet, einfach nur weil sie schön ist? Möglicherweise. Gleichzeitig könnte, und für diese These wäre gerade Zweig ein gutes Beispiel, durch das Tragen von Tracht in diesen Fällen auch eine Art von Heimatverbundenheit ausgedrückt werden, die nicht unbedingt auf Exklusion setzt, sondern als Assimilierung des Wieners, der davor in Zürich gelebt hatte, gedeutet werden kann: Jetzt bin ich hier in Salzburg - jetzt sehe ich aus wie ihr - eine Art Mischung aus Verkleidung als Sommerfrischler und Bekenntnis zu einer bestimmten Form von Bodenständigkeit als konstruierte Heimat, als Verbündung des „Zugereisten“ mit den „Hiesigen“, den Einheimischen. Dass Zweig ebenso wie andere Künstlerinnen und Schriftsteller damit bei der örtlichen Bevölkerung nicht immer gut ankam, ist nicht überraschend, zumal der Antisemitismus und Deutschnationalismus immer stärker wurde. So interpretiert ein Zeitzeuge im Interview Zweigs Kleidung nicht überraschend als „Versuch, sich durch diese Bekleidung nicht als [...] von jüdischer Herkunft erkennen geben zu müssen - also ungestört zu bleiben [...] “(22) - und deutet damit schon ein trauriges späteres Kapitel des Themas Tracht in Salzburg an, das sich abspielte, als Zweig längst im Exil war.

\section{Trachtenverbot in Salzburg}

Im Nationalsozialismus wurden „Andere“, die nicht Teil der „Volksgemeinschaft“ waren, anhand klar sichtbarer Symbole, nämlich Aufnäher, stigmatisiert - das ist bekannt. Weniger bekannt ist möglicherweise, dass in Salzburg nach dem Anschluss Österreichs an NS-Deutschland auch ein ausdrückliches Trachtenverbot für Juden und Jüdinnen ausgesprochen wurde.

21 Cf. Ulrike Kammerhofer-Aggermann, „Von der Trachtenmode zur heiligen vererbten Vätertracht Volk in Tracht ist Macht!'“, in: Marko Feingold (Hg.), Ein ewiges dennoch. 125 Jahre Juden in Salzburg, Wien u. a, 1993, S. 177-190, hier S. 177-184.

22 Interview mit Hofrat Dr. Walter Melcher am 12.5.1993, zitiert nach Alma Scope, „Bühnen der Volkstümlichkeit. Die Bedeutung Salzburgs und der Festspiele für die Trachtenmode“, in: Ulrike Kammerhofer-Aggermann et al. (Hg.), Trachten nicht für Jedermann, Salzburg, 1993, S. 241-260, hier S. 255. 


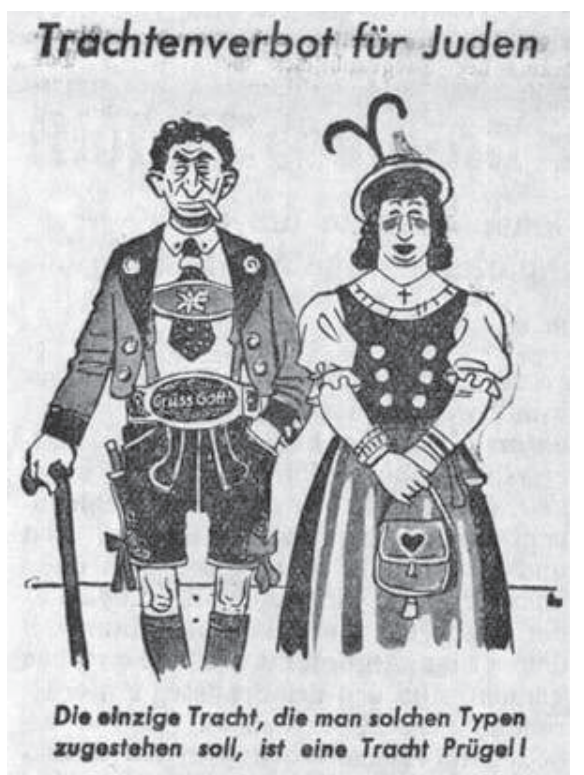

Karikatur aus dem Völkischen Beobachter vom 30. Juni 1938
Dieses Trachtenverbot ist nicht das erste; so untersagte schon 1823 Klemens Metternich die steirische Tracht für Staatsbeamte in Wien, im Jahr 1848 wurden den Slowenen ihre Hüte verboten, auch die ungarische Tracht war im Habsburger-Reich umstritten, wurde sie doch gerade nach dem Sprachdekret Josephs II. und damit der Einführung von Deutsch als Amtssprache demonstrativ im Kampf um mehr Mitsprache bzw. für Unabhängigkeit im Habsburgerreich eingesetzt. Interessant die Strategie von Kaiser Franz Joseph I. und Kaiserin Elisabeth: Die beiden vereinnahmten die Nationalkleidung, indem vor allem Elisabeth demonstrativ ungarische Tracht trug, während Franz Joseph häufig im Steireranzug anzutreffen war. Dadurch und durch die bekannten politischen Zugeständnisse verlor die Kleidung ihren Symbolwert ${ }^{(23)}$ und wurde auch weniger getragen, weil die Abgrenzung durch Tracht vom Wiener Kaiserhaus und die Betonung der Eigenständigkeit einer Gruppe an Sinn verloren hatte.

1938 war die Sachlage jedoch fundamental anders als im 19. Jahrhundert. Das Trachtenverbot, erlassen am 28. Juni 1938, lautete im Wortlaut: „Juden ist im Bereiche der Polizeidirektion Salzburg das öffentliche Tragen von alpenländischen (echten oder unechten) Trachten wie Lederhosen, Joppen, Dirndkleidern, weißen Wadenstutzen, Tirolerhüten usw. verboten." Die Österreichische Alpine, Volks- und Gebirgs-TrachtenZeitung bejubelte den Entschluss, sei doch zum Beispiel das Dirndl „geradezu als ein jüdisches Nationalkostüm“ erschienen ${ }^{(24)}$. Eine Karikatur aus dem Völkischen Beobachter illustriert diese Einstellung: Für die Nationalsozialisten war nur die „echte, rassisch nicht verunreinigte [!] “ Tracht identitätsstiftend.

Durch eine möglichst „deutsche“/,urdeutsche“ Tracht sollte der performative Akt der Zugehörigkeit zur „deutschen“ Volkstumsgemeinschaft vollzogen werden, ein Kollektiv konstruiert werden, in dem das „Andere“ störend war - Ausgrenzung durch Tracht.

Mit der beschriebenen Praxis und der in verschiedenen Arbeiten an anderer Stelle aufgezeigten Trachtenpflege und -erneuerung von Brauchtum im Allgemeinen und Tracht im Besonderen vor allem im österreichischen Raum und im „Sudetenland“ unter den Nationalsozialisten wird natürlich der Höhepunkt des Ausschlussverfahrens

23 Cf. Siegfried Müller, „Die Nationalisierung der Kultur: Das Beispiel der ungarischen Nationaltracht“, in: Ders. (Hg.), Kleider machen Politik. Zur Repräsentation von Nationalstaat und Politik durch Kleidung in Europa vom 18. bis zum 20. Jahrhundert, Oldenburg, 2002, S. 23-31, hier S. 26-29. 
geschildert. Dieser hat, wie am Beginn des Artikels angemerkt, dazu geführt, dass Tracht jahrzehntelang in linken, aufgeklärten Kreisen verpönt war und zum Teil mit Recht als völkisch-deutschtümelnd angesehen wurde.

Interessant erscheint mir deshalb ein vielleicht waghalsiger Sprung ins 21. Jahrhundert: Wenn Tracht so klar ideologisiert und exklusiv war oder ist, wie kann der momentane Trachtenboom erklärt werden? Hat eine völlige Neudeutung oder EntIdeologisierung stattgefunden oder bleibt bei Trachtenträgerinnen und -trägern allgemein oder bei gewissen Gruppierungen, die diese symbolisch einsetzen, bis heute ein schaler Beigeschmack? Darf Tracht guten Gewissens getragen werden, ohne dass dabei vielleicht ungewollte politische Zeichen gesetzt werden, auch bei der politischen Linken, die das im Gegensatz zu konservativen und rechten Parteien großteils vermeidet?

\section{Ent-Ideologisierung und Liberalisierung der Tracht im 21. Jahrhundert?}

Kleidung produziert bis heute Bedeutungen, sie ist ein wesentliches Medium zur Identitätsentwicklung. Kleidung bindet Wertvorstellungen einer Gruppe, repräsentiert nach Max Weber geglaubte Gemeinsamkeiten ${ }^{(25)}$. Vor allem grenzt sie aber als deutlich sichtbares Symbol Gruppen voneinander ab, „andere“ aus, kreiert Räume und damit klar sichtbare Alterität. Im 21. Jahrhundert ist Tracht im weiteren Sinne eine Uniform, eine, die „nicht länger als Diktat, sondern als zeitlich begrenzte Selbstverpflichtung verstanden und praktiziert“(26) wird, die damit auch ganz klar kommuniziert. Dabei halte ich die ganze an früherer Stelle beschriebene Diskussion um die „Echtheit“ von Tracht für gar nicht so relevant: Ein Dirndl der derzeit höchst erfolgreichen österreichischen Modedesignerin Lena Hoschek ist letztlich durch verwendete Formen und Stoffe ebenso Zeichen für Bodenständigkeit und Heimatverbundenheit wie ein für die Musikkapelle geschneidertes Dirndlkleid aus für die Region typischen Stoffen und Mustern. Dieses Beispiel zeigt allerdings den Spagat heutiger Trachtenträgerinnen und Träger auf: Sie stehen gerade in einer stark individualisierten Gesellschaft stärker im Spannungsfeld zwischen Uniformierung, Betonung ihrer Zugehörigkeit zu einer Gemeinschaft und einem hervorgehobenen persönlichen Ausdruck als zum Beispiel Menschen, die Jeans und T-Shirt tragen.

Wenn Menschen Tracht in einer Umgebung tragen, wo sie angeblich nicht hinpasst, findet bei aller Salonfähigkeit bis heute eine Abgrenzung statt: Ein wissenschaftlicher Vortrag an einer Universität im Dirndl ist eher unüblich und würde bei den Zuhörerinnen und Zuhörern vermutlich Zweifel auslösen, ob die Vortragende ernstgenommen werden kann - oder ob sie im besten Fall mit dieser „Verkleidung“ etwas demonstrieren möchte. Wenn in einem Umfeld, in dem sonst Ballkleid und Smoking oder Shorts und Sommerkleider getragen werden, eine Person in Lederhose auftaucht, wird sie je nach sonstigem Verhalten als Sonderling oder besonders kreativ gekleidet wahrgenommen werden. Kommt eine zweite im Janker, einer Jacke aus gewalkter Wolle, hinzu, werden die beiden sofort als Gruppe wahrgenommen. Damit ist Tracht mehr als andere Kleidungsstile gruppenbildend und auch abgrenzend, allein schon deshalb, weil sie in

25 Cf. Max Weber, Wirtschaft und Gesellschaft. Gemeinschaften (Max Weber-Gesamtausgabe, 22, Teilband 1), Tübingen, 2009, S. 44.

26 Cf. Gabriele Mentges et al. (Hg.), Uniformierungen in Bewegung. Vestimentäre Praktiken zwischen Vereinheitlichung, Kostümierung und Maskerade, Münster u. a., 2007, S. 18. 
einigen Regionen nicht üblich ist. Wie ich mehrfach gehört habe, möchten Menschen, die nicht aus typischen Trachtenregionen kommen, zum Beispiel so manche Französin kein Dirndl anziehen, weil ihnen das „nicht authentisch “ vorkäme, „auch wenn es schön“ sei.

Damit ist gerade besagte Französin oder eine andere Person, die die Tracht einer Gruppe trägt, der sie laut deren Homogenitätsvermutung nicht angehört, wieder ein Beleg für diese (Selbst-) Abgrenzung: Mittlerweile wird auch im für Tracht legendären oberösterreichischen Bad Aussee mit den „Zuagroastn“, den Zugereisten, das sind Personen, die erst kurze Zeit - mitunter aber auch schon über eine Generation lang dort leben - weitaus gnädiger umgegangen, wenn sie in echter Ausseer Tracht zu sehen sind. Ein Schmunzeln über die „Verkleidung“ kann aber durchaus vorkommen.

Ebenso sind die Zeiten, konkret die 60er Jahre, in denen der schwarze amerikanische Jazzmusiker Billy Mo mit dem Schlager „Ich kauf mir lieber einen Tirolerhut“ zwar höchst liebenswürdig portraitiert, dabei aber auch eindeutig als Exot dargestellt wurde, vorbei. In Österreich erfolgreich ist 2014 der „Ösi-Bua“ - höchst modern "Cross Dressing“, also Converse Schuhe mit Lederhose tragend, Dreadlocks zum karierten Hemd kombinierend. Doch auch er spielt bis heute mit seiner Herkunft aus Burundi zum bodenständigen oberösterreichischen Akzent, mit einem scheinbaren Widerspruch, der im Jahr 2015 doch eigentlich gar nicht mehr erwähnenswert ist der aber in jedem Zeitungsartikel über ihn angeschnitten und nicht einfach als selbstverständlich angenommen wird ${ }^{(27)}$.

Internationale Trägerinnen und Träger sieht man wohl am häufigsten am Münchner Oktoberfest, das für sich ein interessantes Verhandlungsfeld für Tracht ist, denn in München kommt wieder die Erfindung der Tradition ins Spiel: Auch das Oktoberfest wurde bis Ende der 1970er Jahre eher in Jeans als im Dirndl besucht, vor allem in den letzten zehn Jahren werden die Bilder immer trachtiger ${ }^{(28)}$. Rund um die Jahreszeit sind in München in jedem Billigladen Dirndl zu finden, die auch in jeder Preisklasse und Optik für den Anlass gekauft werden. Einer meiner Studenten - er kommt aus Birmingham - hat mir folgendes erzählt: „Ich bin dorthin gekommen und fand die Leute in Trachten erst blöd. Aber nach ein paar Bier wollte ich auch unbedingt so eine Tracht haben, ich wollte zu diesen Leuten dazugehören“(29). Er betont also bei aller Buntheit und Verschiedenheit der Kleidung, die dort getragen wird, wieder die gruppenbildende Funktion von Tracht.

Gleichzeitig beschweren sich in Interviews „Urbayern“ darüber, dass da Leute unterwegs seien, die „Phantasiekleidung“, ja sogar „Pornodirndl“ trügen, nicht selten ist auch gerade bei den Jüngeren zu hören, dass es doch ein wenig seltsam sei, wenn Australierinnen oder Amerikaner in Tracht herumlaufen würden ${ }^{(30)}$.

27 Siehe dazu Samir Köck, „Ösi-Bua. Aus Afrika mit Lederhose“, Die Presse, 2.4.2012, http://diepresse. com/home/kultur/popco/745589/YouTubeHit-Osi-Bua_Aus-Afrika-mit-Lederhose (Mai 2015).

28 Cf. Manfred Seifert, „Tracht im 21. Jahrhundert. Zum Metabolismus nonverbaler Kommunikation“, in: Ines Keller und Leonore Scholze-Irrlitz (Hg.), Trachten als kulturelles Phänomen der Gegenwart, Bautzen, 2009, S. 9-31, hier S. 24.

29 Gespräch mit Tom Perkins, 25. Oktober 2013.

30 Cf. David Seitz, „Streit um Oktoberfest-Tracht - Dirndl zwischen Tradition und Porno“. Dokumentarfilm, Focus online, https://www.youtube.com/watch?v=33NEx9gX7NU (Oktober 2015). 
Tracht gilt nicht mehr als „Nationaluniform“, ist für Wissenschaftlerinnen wie etwa Thekla Weissengruber bis auf wenige Ausnahmen nicht mehr ideologiebehaftet, keine sinnstiftende Kleidung einer Gruppe mehr ${ }^{(31)}$. Auch Siegfried Müller geht in eine ähnliche Richtung, wenn er meint, dass gerade im „Zeitalter der Überwindung der Nationalstaaten und der Globalisierung" die Tracht als Symbol weitgehend ausgedient habe $^{(32)}$. Vielmehr sei Tracht heute Ausdruck eines postmodernen Identitätsdiskurses ${ }^{(33)}$ : Wir können unsere Identitäten bildlich wechseln, uns verkleiden und am Donnerstag als Punk in der Münchner Innenstadt herumlaufen, am Freitag aber in Tracht am Oktoberfest schunkeln. Wie ich oben zu zeigen versucht habe, glaube ich nicht an eine solche ideologische Befreiung der Tracht. Natürlich ist Kleidung vorerst immer ein Stück Stoff und es kommt auf das Verhalten der Trägerinnen und Träger an. Aber auch im Jahr 2015 kann aus persönlicher Erfahrung wie aus Beobachtung eindeutig festgestellt werden, dass ein Dirndl anders wahrgenommen wird als jede Jeans oder jedes Kleid, dass es auch schnell mit einer (vornehmlich regionalen, im süddeutschen Raum angesiedelten) Gruppe assoziiert wird, und dass „unübliche“ Trägerinnen - die es beim Kleinen Schwarzen ja interessanterweise nicht gibt - immer wieder erklären müssen, wie es denn dazu gekommen sei, dass sie diese Tracht trügen.

So kreiert Tracht bis heute mehr Abgrenzung als Inklusion, weil sich die Geister so sehr an ihr scheiden:

Tschechisch oder deutsch?

„Echte“ Tracht oder „Landhausmode, vulgäre Effekthascherei“(34)?

Verkleidete städtische Doktoren ${ }^{(35)}$ oder „echte“ Bauern?

Nationalistisch-konservative Hinterwäldler oder ironisch-postmodern lediglich zitierendes Cross Dressing, das mit Augenzwinkern und trachtenfremden Elementen darauf hinweist, dass doch eigentlich nur ein Kostüm getragen wird?

Frauen in Lederhosen, Männer in Dirndln? Und wie soll bloß die bärtige österreichische Songcontest-Gewinnerin von 2014, Conchita Wurst eingeordnet werden, wenn sie ein Dirndlkleid trägt?

Da auch Regionaltrachten und deren Anfertigung durch renommierte Häuser wie Tostmann Trachten plötzlich wieder boomen, gehen die Grenzen noch weiter: Soll das niederösterreichische Pittental oder das gleich in der Nähe liegende Schwarzatal repräsentiert werden, trägt die Dame nun ein Wechseldirndl oder das Schneeberglanddirndl - beides in den letzten Jahren kreierte Kleider, die für ebenfalls benachbarte, etwas alpinere Gebiete stehen?

31 Cf. Thekla Weissengruber, Zwischen Pflege und Kommerz. Studien zum Umgang mit Trachten in Österreich nach 1945, Wien, 2004, S. 18.

32 S. Müller, „Die Nationalisierung der Kultur“ (Anm. 23), S. 7.

33 Cf. Heike Frey, „Oktoberfest betrachtet. Zum Bekleidungsverhalten auf der Münchner Wiesn“, in: Keller/Scholze-Irrlitz (Hg.), Trachten als kulturelles Phänomen der Gegenwart (Anm. 28), S. 85-101, hier S. 96. Heike Frey weist allerdings im selben Artikel auch darauf hin, dass „Die Wiesn [...] vehement vermeintlich bayrische Tradition und Heimatgefühle" verhandle, S. 97.

34 Cf. H. Frey, „Oktoberfest betrachtet“ (Anm. 33), S. 95.

35 Ein Zitat von Josef Hanika: „der Herr Doktor gefällt sich dann, einmal einen ,Bauer zu spielen“, J. Hanika, „Neugestaltung der Trachten im Sudetengau“ (Anm. 16), S. 234. 
Mit der Ethnologin Simone Egger halte ich für Tracht im 21. Jahrhundert also keineswegs für ent-ideologisiert, sondern für einen Ausdruck für eine Sehnsucht nach Traditionen, nach langlebigeren Werten und durchaus auch für einen Regionalismus, der einer globalisierten Welt entgegensteht. Gemeinsam mit anderen Trends wie dem Essen heimischer Produkte, am besten dem Züchten von Hühnern im eigenen Garten, dem Stricken von Babykleidung und einer Renaissance der Volksmusik drückt er eine Rückbesinnung auf eine klar definierte kulturelle Identität aus. Der Heimatpfleger Stefan Hirsch bringt die Problematik auf den Punkt, indem er den derzeitigen Trachtenboom mit dem „mangelnden Wissen der vielen Trachtenbegeisterten über das“ begründet, „was sie tragen“. Er fährt fort: „Der eigentliche Grund ist aber sicherlich das Bedürfnis, sich Sehnsuchts- und Gegenwelten zu gönnen, um wenigstens temporär den Zwängen der technischen Zivilisationswelten mit dem Flair scheinbar heiler Ländlichkeit zu entkommen“(36).

Dabei ist diese Suche nach Heimat in weiten Kreisen dezidiert nicht exklusiv, sondern will, wie es auch die für verschiedenste Stile offene, seit den 80er Jahren des 20. Jahrhunderts sehr populäre Volxmusik im süddeutschen Raum zeigt, neue Elemente einbeziehen und mit ihnen auch bildlich spielen. Gleichzeitig zeugt die mehrfach angesprochene Mode von Regional- und sogar Berufstrachten auch von einem gewissen Gemeinschaftsbedürfnis, das offenbar wieder einer individualisierten Welt gegenübersteht: So werden zum Beispiel verschiedenste günstig produzierte Kleider als „Original“ Mohndirndl feilgeboten und versprechen Abnehmerinnen und Abnehmern durchaus eben nicht nur Schönheit durch Stoff und das Design, sondern suggerieren Echtheit, Heimat, konstruierte Tradition mit dazu.

Natürlich findet gleichzeitig in mehreren europäischen Ländern ein Erstarken nationalistischer Bewegungen statt, das auch in diesem Kontext nicht außer Acht gelassen werden darf. Das zeigen politische Demonstrationen eines Sean Connery in Schottland im Kilt ebenso wie Auftritte von ungarischen rechtsextremen Politikern in Nationaltracht, aber auch regionale „Mia san mia“-Auftritte im deutschsprachigen Raum. Das „Wir sind wir“ bildet dabei eine bayrisch-österreichische Formel für ein selbstbewusstes Herausstreichen regionaler Herkunft. Gleichzeitig wird die Demonstration nationaler Identität bei deutschsprachigen Vertriebenen in den letzten Jahren schwächer, wie auch die Sudetendeutsche Landsmannschaft zeigt, indem sie im Jahr 2015 endlich auf Entschädigungsansprüche verzichtet und ihren Diskurs öffnet: Die Inhalte der Treffen scheinen langsam einem neuen Geschichtsverständnis zu weichen, wie unter anderem die Einladung Martin Dušeks zum 64. Sudetendeutschen Tag zeigt - wodurch wiederum eine Gegenbewegung zum neuen Nationalismus aufgezeichnet wird.

Auch die vielerorts gefeierte „Rückkehr der Weiblichkeit“, eine neue Zuschaustellung weiblicher Reize, spielt meiner Ansicht nach beim Trachtenboom mit. Allerdings muss die Gender-Perspektive verbunden mit der Frage, wer nun ein Dirndl gut ausfüllen könnte oder das möchte und welche Zeichen durch Rituale wie das Zuschaustellen von Brüsten eine moderne Gesellschaft aussendet, an anderer Stelle genauer erörtert werden.

36 H. Schмidt, „Was Sie schon immer über Tracht wissen wollten“ (Anm. 11). 


\section{Bewusster und entspannter Umgang mit Tracht führt zu deren Entideologisierung}

Ein kurzes Fazit: Die Tracht ist definitiv offener geworden, Sinnbild bleibt sie weiterhin - nicht oder nur zu einem sehr kleinen Teil für die Blut- und Boden-Ideologie

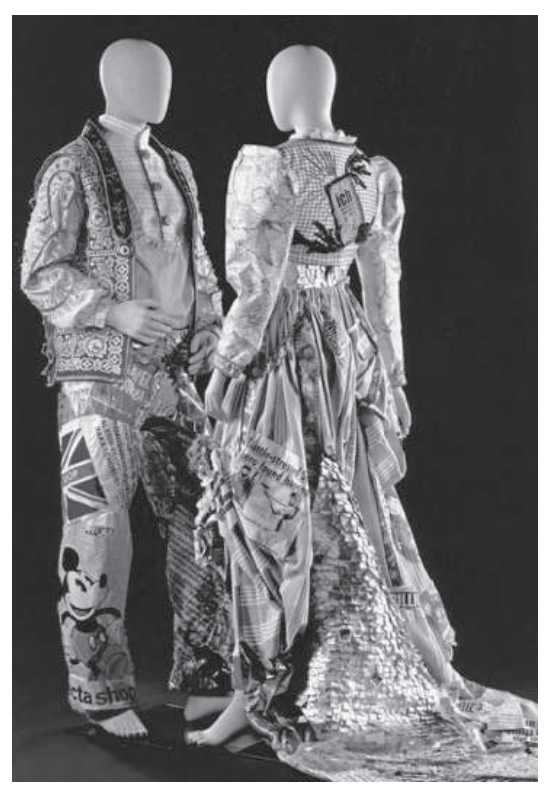

Die Europäische Tracht des Designers Stefan Hann im Museum Europäischer Kulturen in Berlin (mit freundlicher Genehmigung des Museums Europäischer Kulturen) der 30er Jahre, aber vielleicht wie damals in Salzburg einerseits für einen durchaus nationalistisch-chauvinistischen Zugang und andererseits für ein fröhliches Bekenntnis zur gewählten Heimat, selbst und gerade wenn eine Ironisierung durch Cross Dressing stattfindet. Denn gerade dann, wenn Tracht sich selbst nicht zu ernst nimmt und den Trägerinnen und Trägern die Konstruiertheit und abgrenzende Funktion dieser Kleidungsform klar ist, kann mit dem Stück materieller Kultur am besten gespielt werden, weshalb Aufklärung über die Geschichte dieses Stücks konstruierter Heimat zentral ist.

Interessant ist in dem Zusammenhang eine Kreation des Berliner Museums Europäischer Kulturen: Der Modedesigner Stefan Hann hat versucht, das Thema Tracht neu zu interpretieren und eine europäische „Tracht“ zu kreieren, die im Jahr 2013 die gemeinsamen Werte repräsentieren könnte. Bewusst werden hier sowohl Regionalismus als auch Echtheit ausgespart - hier das Foto dieser neuen „Tracht“ - so sie eine ist.

\section{Literatur}

Benedict Anderson, Imagined Communities. Reflections on the Origin and Spread of Nationalism, London, 1983.

Martin DušEK, Mein kroj. Dokumentarfilm, Prag, 2011, http://www.ceskatelevize.cz/ porady/10318067925-mein-kroj/ (Mai 2015).

Simone EgGer, Phänomen Wiesntracht. Identitätspraxen einer urbanen Gesellschaft, München, 2008.

Elisabeth FendL, „Die Politisierung der Tracht im Egerland des frühen 20. Jahrhunderts. Eine Projektskizze", in: Heidrun Alzheimer et al. (Hg.), Jahrbuch für europäische Ethnologie, Paderborn u. a., 2009, S. 69-80.

Heike Frey, „Oktoberfest betrachtet. Zum Bekleidungsverhalten auf der Münchner Wiesn“, in: Ines Keller und Leonore Scholze-Ir Rlitz (Hg.), Trachten als kulturelles Phänomen der Gegenwart, Bautzen, 2009 (Schriften des Sorbischen Instituts, 49), S. 85-101.

Ernest Gellner, Nations and Nationalism, Oxford, 1983.

Hans Peter Hahn, Materielle Kultur. Eine Einführung, Berlin, 2005.

Josef HaniKa, „Neugestaltung der Trachten im Sudetengau“, Deutsche Volkskunde, 3 (1939), S. 234-240.

Josef Hanika, Sudetendeutsche Volkstrachten, Reichenberg, 1937 (Beiträge zur sudetendeutschen Volkskunde, 22). 
Eric Hobsbawm und Terence Ranger, The Invention of Tradition, Cambridge, 2002.

Ulrike Kammerhofer-Aggermann, „Von der Trachtenmode zur heiligen vererbten Vätertracht - ,Volk in Tracht ist Macht! “', in: Marko FeINGold (Hg.), Ein ewiges dennoch. 125 Jahre Juden in Salzburg, Wien u. a., 1993, S. 177-190.

Rudi Keller, Tracht am Oberrhein, Strasbourg, 1942.

Samir KöcK, „Ösi-Bua. Aus Afrika mit Lederhose“, Die Presse, 2.4.2012, http://diepresse.com/ home/kultur/popco/745589/YouTubeHit-Osi-Bua_Aus-Afrika-mit-Lederhose (Mai 2015).

Gertrud Lehnert, „Mode und Moderne“, in: Gabriele Mentges (Hg.), Kulturanthropologie des Textilen, Berlin, 2005, S. 251-263.

Jean-Pierre Lethuillier, Les costumes régionaux entre mémoire et histoire, Rennes, 2009.

Petr LozoviuK, „Die sudetendeutsche Volkskunde in den 1930er- und 1940er-Jahren. Von der Herkunftsfrage der kulturellen Erscheinungen zur ,Rassenkunde“ in der ,Volksforschung “", Volkskunde in Sachsen, 19 (2007), S. 199-214.

Elke Mehnert (Hg.), Landschaften der Erinnerung. Flucht und Vertreibung aus deutscher, polnischer und tschechischer Sicht, Frankfurt, 2001 (Studien zur Reiseliteratur- und Imagologieforschung, 5).

Gabriele Mentges et al. (Hg.), Uniformierungen in Bewegung. Vestimentäre Praktiken zwischen Vereinheitlichung, Kostümierung und Maskerade, Münster u. a., 2007 (Schriftenreihe Museum Europäischer Kulturen, 4).

Siegfried MülLER, „Die Nationalisierung der Kultur: Das Beispiel der ungarischen Nationaltracht", in: Ders. (Hg.), Kleider machen Politik. Zur Repräsentation von Nationalstaat und Politik durch Kleidung in Europa vom 18. bis zum 20. Jahrhundert, Oldenburg, 2002 (Kataloge des Landesmuseums Oldenburg, 19), S. 23-31.

Peter Rosegger, „Künstliches Volksthum“, Heimgarten, 19 (1895).

Fritz Rumpf, Der Mensch und seine Tracht ihrem Wesen nach geschildert, Berlin, 1905.

Petra SCHeIPER, Textile Metamorphosen als Ausdruck gesellschaftlichen Wandels. Das Bekleidungsverhalten junger Männer und Frauen als Phänomen der Grenzverschiebung von Sexund Gender-Identitäten, Hannover, 2006.

Heidi Schмidт, „Was Sie schon immer über Tracht wissen wollten“, Interview mit Stefan Hirsch, Alpenmagazin, o. J, http://www.alpenmagazin.org/index.php/gesellschaft/100deutschland26/702-was-sie-schon-immer-ueber-trachten-wissen-wollten (Mai 2015).

Alma SCOPE, „Bühnen der Volkstümlichkeit. Die Bedeutung Salzburgs und der Festspiele für die Trachtenmode“, in: Ulrike Kammerhofer-Aggermann et al. (Hg.), Trachten nicht für Jedermann, Salzburg, 1993 (Salzburger Beiträge zur Volkskunde, 6), S. 241-260.

Manfred SEIfERT, „Tracht im 21. Jahrhundert. Zum Metabolismus nonverbaler Kommunikation“, in: Ines Keller und Leonore Scholze-Irrlitz (Hg.), Trachten als kulturelles Phänomen der Gegenwart, Bautzen, 2009 (Schriften des Sorbischen Instituts, 49), S. 9-31.

David Seitz, „Streit um Oktoberfest-Tracht - Dirndl zwischen Tradition und Porno“. Dokumentarfilm, Focus online, https://www.youtube.com/watch?v=33NEx9gX7NU (Oktober 2015).

Elisabeth Tietmey er et al., Die Sprache der Dinge. Kulturwissenschaftliche Perspektiven auf die materielle Kultur, Münster u. a., 2010.

Max Weber, Wirtschaft und Gesellschaft. Gemeinschaften (Studienausgabe der Max WeberGesamtausgabe, 22, Teilband 1), Tübingen, 2009.

Tobias Weger, „Die,Volksgruppe im Exil'? Sudetendeutsche Politik nach 1945“, in: Hans Henning HaHN (Hg.), Hundert Jahre sudetendeutsche Geschichte. Eine völkische Bewegung in drei Staaten, Frankfurt, 2007 (Die Deutschen und das östliche Europa, Studien und Quellen, 1), S. 277-301.

Tobias Weger, „,Völkische‘ Wissenschaft zwischen Prag, Eger und München. Das Beispiel Josef Hanika“, in: Christiane Brenner et al. (Hg.), Geschichtsschreibung zu den böhmischen Ländern im 20. Jahrhundert, München, 2006 (Bad Wiesseer Tagungen des Collegium Carolinum, 28), S. 177-208. 
Tobias Weger, „Volkstumskampf“ ohne Ende? Sudetendeutsche Organisationen,1945-1955, Frankfurt u. a., 2008 (Die Deutschen und das östliche Europa, 2).

Hans-Ulrich Wehler, Nationalismus. Geschichte, Formen, Folgen, München, 2007.

Thekla Weissengruber, Zwischen Pflege und Kommerz. Studien zum Umgang mit Trachten in Österreich nach 1945, Wien, 2004.

Marco ZimmermanN, „Ein satirischer Beitrag zur Identitätssuche: Der Dokumentarfilm Mein kroj“. Radio Praha, 2012, http://www.radio.cz/de/rubrik/kultur/ein-satirischer-beitrag-zuridentitaetssuche-der-dokumentarfilm-mein-kroj (Mai 2015).

Martin ZÜCKERT, „Die Volkskunde als Nachbardisziplin der, sudetendeutschen' Geschichtsschreibung. Gegenseitige Beeinflussung und parallele Forschungsinteressen“, in: Stefan ALBRECHT et al. (Hg.), Die „sudetendeutsche Geschichtsschreibung“ 1918-1960. Zur Vorgeschichte und Gründung der Historischen Kommission der Sudetenländer, München, 2008 (= Veröffentlichungen des Collegium Carolinum, 114), S. 183-199.

„Landsmannschaft verzichtet auf,Wiedergewinnung der Heimat“", Die Zeit vom 1. März 2015, http://www.zeit.de/politik/deutschland/2015-03/sudetendeutsche-landsmannschaft-heimat (Mai 2015).

Gespräch mit Martin Dušek am 4. November 2013.

Gespräch mit Tom Perkins am 25. Oktober 2013.

\section{Zusammenfassung}

Im Artikel „Konstruierte Heimat" beschäftigt sich die Autorin mit der Frage, wie sehr Tracht bis heute abgrenzend bzw. exklusiv wirkt und untersucht dafür den Film Mein kroj, auf Deutsch „Meine Tracht“ von Martin Dušek. In seinem Film zeigt der 1978 geborene tschechische Filmemacher geschickt sowohl die Konstruiertheit als auch die ideologische Aufladung von Tracht in ehemals deutschsprachigen Gebieten in Tschechien auf und persifliert die auf beiden Seiten der Grenze mithilfe von Kleidung konstruierte Heimat mit seinem „Kroj", seiner ganz persönlichen Tracht, die aus Familienerinnerungen besteht. Ausgehend von dieser filmischen Dekonstruktion versucht die Autorin an den Beispielen Salzburg in den 30er Jahren und dem Trachtenboom der Jahrtausendwende aufzuzeigen, dass Tracht mit ideologischer Bedeutung aufgeladen bleibt. Dies sollte Trägerinnen und Trägern bewusst sein, sodass der ausschließende Charakter von Kleidungsstücken, die die Tradition zitieren, aufgebrochen werden kann.

\section{Résumé}

L'auteure analyse le film Mein kroj (Mon costume folklorique) de Martin Dušek pour étudier jusqu'à quel point les vêtements folkloriques ont une signification de démarcation voire d'exclusion. Ce cinéaste, né en 1978 en République tchèque, démontre dans son film non seulement l'origine de la tradition liée aux vêtements folkloriques mais aussi la connotation idéologique de ces costumes dans les régions jadis germanophones de la République tchèque. Avec son propre "Kroj" - des vêtements folkloriques qu'il a bricolés tout seul - il tourne en ridicule la construction d'une Heimat à travers les vêtements des deux côtés de la frontière germano-tchèque. Partant de cette déconstruction filmique, l'auteure essaie de montrer, notamment en employant les exemples de Salzbourg dans les années 30 et le boom des vêtements folkloriques à la fin du millénaire, que les costumes régionaux restent chargés d'une certaine idéologie. Les porteurs de ces costumes devraient être conscients de ce fait, afin que le caractère exclusif de ce genre de vêtements puisse être combattu. 